\title{
Associations of diet quality with cognition in children - the Physical Activity and Nutrition in Children Study
}

\author{
Eero A. Haapala ${ }^{1 *}$, Aino-Maija Eloranta ${ }^{1,2}$, Taisa Venäläinen ${ }^{1}$, Ursula Schwab ${ }^{2,3}$, Virpi $\operatorname{Lindi}^{1}$ and \\ Timo A. Lakka ${ }^{1,4,5}$ \\ ${ }^{1}$ School of Medicine, Institute of Biomedicine, University of Eastern Finland, PO Box 1627, FI-70211 Kuopio, Finland \\ ${ }^{2}$ Institute of Public Health and Clinical Nutrition, University of Eastern Finland, Kuopio, Finland \\ ${ }^{3}$ Institute of Clinical Medicine, Internal Medicine, Kuopio University Hospital, Kuopio, Finland \\ ${ }^{4}$ Department of Clinical Physiology and Nuclear Medicine, Kuopio University Hospital and University of Eastern Finland, \\ Kuopio, Finland \\ ${ }^{5}$ Kuopio Research Institute of Exercise Medicine, Kuopio, Finland \\ (Submitted 7 January 2015 - Final revision received 14 April 2015-Accepted 15 April 2015 - First published online 14 August 2015)
}

\section{Abstract}

Evidence on the associations of dietary patterns with cognition in children is limited. Therefore, we investigated the associations of the Baltic Sea Diet Score (BSDS) and the Dietary Approaches to Stop Hypertension (DASH) score with cognition in children. The present cross-sectional study sample included 428 children aged $6-8$ years ( 216 boys and 212 girls). The BSDS and the DASH score were calculated using data from $4 \mathrm{~d}$ food records, higher scores indicating better diet quality. Cognition was assessed by the Raven's Coloured Progressive Matrices (CPM) score, a higher score indicating better cognition. Among all children, the BSDS (standardised regression coefficient $\beta=0 \cdot 122, P=0 \cdot 012)$ and the DASH score $(\beta=0 \cdot 121, P=0.015)$ were directly associated with the Raven's CPM score. Among boys, a lower BSDS $(\beta=0.244, P<0.001)$ and a lower DASH score $(\beta=0.202, P=0.003)$ were related to a lower Raven's CPM score. Boys in the lowest quartile of the BSDS (22.5 v. 25.3, $P=0.029)$ and the DASH score (22.4 $v .25 \cdot 7, P=0 \cdot 008)$ had a lower Raven's CPM score than those in the highest quartile of the corresponding score. Among girls, the BSDS or the DASH score were not associated with cognition. In conclusion, a poorer diet quality was associated with worse cognition in children, and the relationship was stronger in boys than in girls.

Key words: Diet quality: Diet: Children: Brain: Cognition

Adequate nutrition is the foundation of normal physical and cognitive development in children ${ }^{(1)}$. Undernourishment and low availability of nutritionally adequate and safe foods have been found to decrease cognitive functions in children ${ }^{(2,3)}$. Nevertheless, abundance of foods containing lots of sucrose and saturated fat has been linked to cognitive decline in adults $^{(4)}$. In fact, unhealthy food choices may be a more important determinant of decreased cognition than undernourishment among children in developed countries, including Finland.

Evidence on the associations of dietary factors with cognition in children is limited. A higher intake of SFA and trans-fatty acids has been linked to poorer working memory ${ }^{(5,6)}$, a lower intake of dietary $n-3$ fatty acids has been associated with worse hippocampus-dependent memory ${ }^{(5)}$, and a lower intake of dietary fibre has been related to poorer cognitive control in children ${ }^{(7)}$. These studies have suggested that some nutrients have been associated with a family of effortful top-down cognitive processes called executive functions that involve inhibition, working memory and mental flexibility ${ }^{(8)}$. However, it is also important to investigate the associations of overall dietary patterns with cognition in children, because the health effects of dietary patterns can be more easily translated to public health messaging since people eat and buy foods rather than nutrients, and because nutrients do not exist in isolation and are strongly interrelated $^{(9,10)}$

The Dietary Approaches to Stop Hypertension (DASH) score, which was originally developed for the prevention of hypertension in adults ${ }^{(11)}$, can be easily adopted in most countries. A lower DASH score has been linked to poorer cognitive functions in older adults ${ }^{(12)}$; however, there are no

Abbreviations: BSDS, Baltic Sea Diet Score; CPM, Coloured Progressive Matrices; DASH, Dietary Approaches to Stop Hypertension; PANIC, Physical Activity and Nutrition in Children.

*Corresponding author: Dr E. A. Haapala, fax +35817 162 131, email eero.haapala@uef.fi 
reports on the associations of the DASH score with cognition in children. Poorer adherence to the Mediterranean style diet has not only been related to worse cognition and more rapid cognitive decline among older adults ${ }^{(10,13)}$ but also to worse academic achievement in children ${ }^{(14)}$. However, the Mediterranean Diet Score may not be easily adopted by other countries because of differences in food cultures. Therefore, a Finnish research group recently created the Baltic Sea Diet Score (BSDS) to assess diet quality in the Nordic countries ${ }^{(15)}$. However, there are no reports on the associations of the BSDS with cognition in adults or children.

There is some evidence on the direct associations of diet quality indices with cognition in older adults; however, little is known about these relationships in children ${ }^{(16)}$. We therefore investigated the associations of the BSDS and the DASH score, which can be easily adopted in other countries, with cognition in a population sample of Finnish children.

\section{Methods}

The present analyses are based on the baseline data from the Physical Activity and Nutrition in Children (PANIC) Study, which is an ongoing physical activity and diet intervention study in a population sample of primary school children from the city of Kuopio, Finland ${ }^{(17)}$. Altogether, 736 children aged 6-8 years were invited to participate in the baseline examinations between 2007 and 2009, as described earlier ${ }^{(17)}$. Of the invited children, $512(70 \%)$ participated. The participants did not differ in sex distribution, age or BMI standard deviation score from other children of the same age whose data were obtained from school health examinations (data not shown). The present study sample included 428 children (216 boys and 212 girls) for whom we had complete data on variables used in the analyses. Children who were excluded from the study because of incomplete data had lower levels of total physical activity and a lower education and income of the parents than the included children $(P<0.05)$. The PANIC Study protocol was approved by the Research Ethics Committee of the Hospital District of Northern Savo. All children and their parents gave written informed consent. The study was registered at Clinicaltrials.gov (NCT01803776).

\section{Assessment of dietary factors}

We assessed food consumption and nutrient intake by food records administered by the parents on four predefined consecutive days, including either two weekdays and two weekend days (99.5\% of children) or three weekdays and one weekend day $(0.5 \% \text { of children })^{(17)}$. A clinical nutritionist instructed the parents to record all food and drinks using household or other measures, such as tablespoons, decilitres and centimetres. The parents were also instructed to ask their child about food eaten outside home. Moreover, a clinical nutritionist asked about details of menus and recipes of food served at schools and afternoon day care from the catering company that provided the food for the schools. A clinical nutritionist used all this information and also a picture booklet of portion sizes ${ }^{(18)}$ when reviewing and completing the food records at return, if needed. We analysed the food records and calculated total energy intake using the Micro Nutrica ${ }^{\circledR}$ dietary analysis software, version 2.5 (The Social Insurance Institution of Finland), that utilises Finnish and international data on nutrient intakes of foods ${ }^{(19)}$. We computed the BSDS and the DASH score as described in Table 1. The agreement between the BSDS and the DASH score was moderate $(\kappa$ coefficient $=0.324, P<0.001)$, suggesting that these indices of diet quality represent partly different entities of healthy diet.

We assessed the number of meals per $d$ based on data from the food records. We classified breakfast, lunch and dinner as meals and all eating and drinking occasions between the meals as snacks. We categorised the children as those who had eaten all meals daily and those who had skipped any of the meals.

We assessed eating behaviour by the Children's Eating Behaviour Questionnaire administrated by the parents that has been validated ${ }^{(20)}$ and translated into Finnish earlier. The thirty-five questions of the questionnaire represented eight categories of eating behaviour, including food approach (enjoyment of food, food responsiveness, emotional overeating, desire to drink) and food avoidance (satiety responsiveness, slowness in eating, emotional undereating

Table 1. Construction of the Baltic Sea Diet Score (BSDS) and the Dietary Approaches to Stop Hypertension (DASH) score in the present study

\begin{tabular}{|c|c|c|}
\hline & Components & Scoring \\
\hline \multirow[t]{8}{*}{ BSDS $^{(15)}$} & Fruit and berries $(\mathrm{g} / \mathrm{d})$ & Quartile $1=0$, Quartile $2=1$, Quartile $3=2$, Quartile $4=3$ \\
\hline & Vegetables (legumes included, potatoes excluded) $(\mathrm{g} / \mathrm{d})$ & Quartile $1=0$, Quartile $2=1$, Quartile $3=2$, Quartile $4=3$ \\
\hline & High-fibre $(\geq 5 \%)$ grain products $(\mathrm{g} / \mathrm{d})$ & Quartile $1=0$, Quartile 2 $=1$, Quartile $3=2$, Quartile $4=3$ \\
\hline & Low-fat $(<1 \%)$ milk $(\mathrm{g} / \mathrm{d})$ & Quartile $1=0$, Quartile $2=1$, Quartile $3=2$, Quartile $4=3$ \\
\hline & Fish $(g / d)$ & Quartile $1=0$, Quartile $2=1$, Quartile $3=2$, Quartile $4=3$ \\
\hline & Red meat and sausage $(\mathrm{g} / \mathrm{d})$ & Quartile $1=3$, Quartile $2=2$, Quartile $3=1$, Quartile $4=0$ \\
\hline & Ratio of PUFA:SFA & Quartile $1=0$, Quartile $2=1$, Quartile $3=2$, Quartile $4=3$ \\
\hline & Total fat intake (\% energy intake) & Quartile $1=3$, Quartile $2=2$, Quartile $3=1$, Quartile $4=0$ \\
\hline \multirow[t]{7}{*}{ DASH score ${ }^{(31)}$} & Fruit and fruit juices $(\mathrm{g} / \mathrm{d})$ & Quintile $1=1$, Quintile $2=2$, Quintile $3=3$, Quintile $4=4$, Quintile $5=5$ \\
\hline & Vegetables (potatoes excluded) $(\mathrm{g} / \mathrm{d})$ & Quintile $1=1$, Quintile $2=2$, Quintile $3=3$, Quintile $4=4$, Quintile $5=5$ \\
\hline & High-fibre ( $\geq 5 \%$ ) grain products $(\mathrm{g} / \mathrm{d})$ & Quintile $1=1$, Quintile $2=2$, Quintile $3=3$, Quintile $4=4$, Quintile $5=5$ \\
\hline & Low-fat $(<1 \%)$ milk and sour milk products $(\mathrm{g} / \mathrm{d})$ & Quintile $1=1$, Quintile $2=2$, Quintile $3=3$, Quintile $4=4$, Quintile $5=5$ \\
\hline & Red meat and sausage $(\mathrm{g} / \mathrm{d})$ & Quintile $1=5$, Quintile $2=4$, Quintile $3=3$, Quintile $4=2$, Quintile $5=1$ \\
\hline & Sugar-sweetened beverages $(\mathrm{g} / \mathrm{d})$ & Quintile $1=5$, Quintile $2=4$, Quintile $3=3$, Quintile $4=2$, Quintile $5=1$ \\
\hline & $\mathrm{Na}$ intake $(\mathrm{mg})$ & Quintile $1=5$, Quintile $2=4$, Quintile $3=3$, Quintile $4=2$, Quintile $5=1$ \\
\hline
\end{tabular}


and food fussiness). Each question offered options from never to always on a five-point Likert scale, and the means of responses of each category were calculated and used in the analyses.

\section{Assessment of cognition}

We used Raven's Coloured Progressive Matrices (CPM) to assess non-verbal reasoning ${ }^{(21)}$. One trained researcher administered these assessments. Raven's CPM includes thirty-six large figures with a part missing. The children were asked to select the correct part that completes the figure from six alternatives presented beneath the large figure. Raven's CPM requires the ability to find similarities, differences and discrete patterns, does not depend on acquired knowledge or language skills ${ }^{(21)}$, and has been suggested to represent all-core components of executive functions ${ }^{(8)}$. The Raven's CPM score was the number of correct answers, ranging from 0 to 36 .

\section{Other assessments}

We assessed cardiovascular performance using a maximal exercise test with an electromagnetically braked Ergoselect $200 \mathrm{~K}^{\circledR}$ cycle ergometer (Ergoline). We used maximal workload per lean body mass as a measure of cardiovascular performance $^{(22)}$. We used the sum of $Z$-scores for the $50 \mathrm{~m}$ agility shuttle run test time (inverse), errors in the flamingo balance test (inverse), and the number of cubes moved in the box and block test as a measure of motor performance ${ }^{(23)}$. We assessed total physical activity and total screen-based sedentary behaviour, including the time spent watching television and videos, using computer and playing video games, and using mobile phone and playing mobile games by the PANIC Physical Activity Questionnaire administered by the parents together with their children at home ${ }^{(24,25)}$. We calculated BMI standard deviation score using Finnish age- and sex-specific reference values ${ }^{(26)}$ and defined the prevalence of overweight and obesity using the cut-off values provided by cole et $a l^{(27)}$. We measured body fat percentage and lean body mass by the Lunar ${ }^{\circledR}$ dual-energy X-ray absorptiometry device (Lunar Prodigy Advance; GE Medical Systems) ${ }^{(28)}$. The research physician assessed pubertal status using the five-stage criteria described by Tanner ${ }^{(29)}$. The boys were defined as having entered clinical puberty, if their testicular volume assessed by an orchidometer was $>3 \mathrm{ml}$ (Stage $\geq 2$ ). The girls were defined having entered puberty, if their breast development had started (Stage $\geq 2$ ). We also used the child's current height as a percentage of predicted adult height as a measure of maturity ${ }^{(30)}$. The parents were also asked to report medically diagnosed development disorders of their child, such as attention deficit hyperactivity disorder, dysphasia or delayed neurological development.

The parents were asked to report their annual household income, that was categorised as $\leq 30000 €, 30001-60000 €$ and $>60000 €$ for the analyses. The parents were also asked to report their highest completed or ongoing educational degrees (e.g. vocational school or less, polytechnic and university) and the degree of the more educated parent was used in the analyses.

\section{Statistical analyses}

We performed all data analyses using the SPSS Statistics software, version 21.0 (IBM Corporation). We compared basic characteristics between boys and girls using the Student's $t$ test, the Mann-Whitney's $U$ test, and the $\chi^{2}$ test. The associations of the BSDS and the DASH score and their components with the Raven's CPM score were investigated using the multivariate linear regression analysis. Age, sex, parental education and household income were entered into the model in Block 1 , and the BSDS or the DASH score were forced into the model in Block 2. In the analysis for the components of the BSDS and the DASH score, age, sex, parental education and household income were forced into the models in Block 1, and the components of the BSDS or the DASH score were entered stepwise into the models in Block 2. We compared the Raven's CPM score among children in the quartiles of the BSDS, the DASH score and their components using General Linear Models adjusted for age, sex, parental education and household income. The only exceptions were the components of the DASH score, for which we used quintiles according to Fung et al. ${ }^{(31)}$ (Table 1). The consumption of fish was divided to thirds, because of a large number of children reporting no fish consumption. If the associations of the BSDS or the DASH score with cognition or the differences in cognition among the groups of the BSDS or the DASH score were statistically significant after adjustment for age, sex, parental education and household income, the data were additionally adjusted for total physical activity, total screenbased sedentary behaviour, cardiovascular performance, motor performance, body fat percentage, clinical puberty, the current height as a percentage of predicted adult height or development disorders. We selected covariates for the analyses based on the evidence that the associations of diet quality with cognition may be confounded by socio-economic status $^{(32)}$, physical activity ${ }^{(33)}$, physical performance ${ }^{(34)}$, $\operatorname{adiposity}^{(35)}$, maturity ${ }^{(36)}$ and development disorders ${ }^{(37)}$. We finally adjusted the data for total energy intake, skipping meals or eating behaviours to study whether other dietary factors affected the associations of the BSDS or the DASH score with the Raven's CPM score.

Because the associations of the BSDS and the DASH score with the Raven's CPM score were consistently stronger in boys than in girls and because sex statistically significantly modified the association between the BSDS and the Raven's CPM score ( $P=0.045$ for interaction), we also conducted the analyses separately among boys and girls.

\section{Results}

\section{Basic characteristics}

The boys had attained a smaller proportion of their predicted adult height, had a lower body fat percentage, a poorer motor performance and a better cardiovascular performance, were 
physically more active, had more screen-based sedentary behaviour, came more often from families with a parent with university degree, skipped less often meals and had a higher total energy intake than the girls (Table 2). The boys also had a lower DASH score, a higher consumption of red meat and sausages and a higher intake of $\mathrm{Na}$ compared with the girls (Table 2).

\section{Associations of Baltic Sea Diet Score, Dietary Approaches to Stop Hypertension score and their components with Raven's Coloured Progressive Matrices score}

Among all children, a lower BSDS and DASH score were related to a lower Raven's CPM score after adjustment for age, sex, parental education and household income (Table 3). Of the components of the BSDS, a higher consumption of red meat and sausages was associated with a lower Raven's CPM score after these adjustments (Table 3). Of the components of the DASH score, a lower consumption of fruit and fruit juices and a higher consumption of red meat and sausages were related to a lower Raven's CPM score after adjustment for age, sex, parental education and household income (Table 3). Further adjustment for total physical activity, total screen-based sedentary behaviour, cardiovascular performance, motor performance, body fat percentage, clinical puberty, the current height as a percentage of predicted adult height, development disorders, total energy intake, skipping meals or eating behaviours had no effect on these associations (data not shown). These associations remained similar when the analyses were performed after excluding twelve children who had development disorders (data not shown).

Table 2. Basic characteristics

(Mean values and standard deviations, medians and interquartile ranges, or percentages ${ }^{*}$ )

\begin{tabular}{|c|c|c|c|c|c|c|c|}
\hline & \multicolumn{2}{|c|}{ All $(n$ 428) } & \multicolumn{2}{|c|}{ Boys $(n 216)$} & \multicolumn{2}{|c|}{ Girls ( $n$ 212) } & \multirow[b]{2}{*}{$P \dagger$} \\
\hline & Mean & SD & Mean & SD & Mean & SD & \\
\hline Age (years) & $7 \cdot 6$ & 0.4 & $7 \cdot 6$ & 0.4 & $7 \cdot 6$ & 0.4 & 0.506 \\
\hline Pre-pubertal (\%) & \multicolumn{2}{|c|}{97.2} & \multicolumn{2}{|c|}{98.6} & \multicolumn{2}{|c|}{95.7} & 0.076 \\
\hline Height as a percentage of predicted adult height & 75.5 & $3 \cdot 8$ & $72 \cdot 1$ & $2 \cdot 6$ & $77 \cdot 1$ & 3.0 & $<0.001$ \\
\hline Body fat percentageł & $18 \cdot 7$ & $10 \cdot 7$ & $15 \cdot 1$ & $10 \cdot 4$ & $20 \cdot 6$ & $9 \cdot 8$ & $<0.001$ \\
\hline BMI standard deviation score & -0.18 & $1 \cdot 1$ & -0.20 & $1 \cdot 1$ & -0.17 & $1 \cdot 1$ & 0.769 \\
\hline Prevalence of overweight and obesity (\%) & \multicolumn{2}{|c|}{$12 \cdot 4$} & \multicolumn{2}{|c|}{10.2} & \multicolumn{2}{|c|}{14.6} & 0.169 \\
\hline Motor performance score & 0.2 & $2 \cdot 6$ & -0.2 & $2 \cdot 7$ & 0.5 & 0.3 & 0.006 \\
\hline Cardiovascular performance (W/kg lean body mass) & $3 \cdot 7$ & 0.5 & 3.8 & 0.5 & 3.6 & 0.5 & $<0.001$ \\
\hline Total physical activity $(\mathrm{min} / \mathrm{d}) \ddagger$ & 109 & $63 \cdot 6$ & 117 & $67 \cdot 5$ & 100 & $61 \cdot 0$ & $<0.001$ \\
\hline Total screen-based sedentary behaviour $(\mathrm{min} / \mathrm{d}) \ddagger$ & $95 \cdot 4$ & $62 \cdot 9$ & 101 & $63 \cdot 2$ & $90 \cdot 0$ & $55 \cdot 7$ & $<0.001$ \\
\hline Parental education (\%) & & & & & & & 0.006 \\
\hline Vocational school or less & \multicolumn{2}{|c|}{$17 \cdot 1$} & \multicolumn{2}{|c|}{$18 \cdot 6$} & \multicolumn{2}{|c|}{$15 \cdot 6$} & \\
\hline Polytechnic & \multicolumn{2}{|c|}{$45 \cdot 7$} & \multicolumn{2}{|c|}{$45 \cdot 7$} & \multicolumn{2}{|c|}{$53 \cdot 3$} & \\
\hline University degree & \multicolumn{2}{|c|}{$37 \cdot 2$} & \multicolumn{2}{|c|}{$43 \cdot 3$} & \multicolumn{2}{|c|}{$31 \cdot 1$} & \\
\hline Household income (\%) & & & & & & & 0.080 \\
\hline$<30001 €$ & \multicolumn{2}{|c|}{19.5} & \multicolumn{2}{|c|}{$16 \cdot 2$} & \multicolumn{2}{|c|}{22.9} & \\
\hline $30001-60000 €$ & \multicolumn{2}{|c|}{41.9} & \multicolumn{2}{|c|}{40.5} & & & \\
\hline$>60000 €$ & & & & & & & \\
\hline Prevalence of development disorders (\%) & & & & & & & 0.085 \\
\hline Skipping meals (\%) & & & & & & & 0.035 \\
\hline Total energy intake & & & & & & & $<0.001$ \\
\hline $\mathrm{kcal} / \mathrm{d}$ & 1641 & 309 & 1732 & 310 & 1548 & 279 & \\
\hline $\mathrm{kJ} / \mathrm{d}$ & 6866 & 1293 & 7247 & 1297 & 6477 & 1167 & \\
\hline Baltic Sea Diet Score & 11.8 & 4.4 & 11.5 & 4.5 & $12 \cdot 1$ & $4 \cdot 2$ & 0.118 \\
\hline Fruit and berries $(g / d) \ddagger$ & $92 \cdot 7$ & $97 \cdot 3$ & 88.3 & 105 & 95.4 & 471 & 0.101 \\
\hline Vegetables (legumes included, potatoes excluded) (g/d) & $93 \cdot 7$ & $72 \cdot 8$ & $88 \cdot 6$ & $75 \cdot 2$ & $95 \cdot 9$ & $70 \cdot 6$ & 0.435 \\
\hline High-fibre $(\geq 5 \%)$ grain products $(\mathrm{g} / \mathrm{d})$ & $57 \cdot 3$ & $49 \cdot 0$ & $60 \cdot 1$ & 48.0 & $55 \cdot 1$ & $48 \cdot 7$ & 0.247 \\
\hline Low-fat $(<1 \%)$ milk $(\mathrm{g} / \mathrm{d}) \ddagger$ & 371 & 513 & 417 & 542 & 355 & 497 & 0.132 \\
\hline Fish $(g / d) \ddagger$ & 6.5 & $25 \cdot 7$ & $8 \cdot 1$ & $28 \cdot 2$ & $5 \cdot 0$ & $24 \cdot 3$ & 0.342 \\
\hline Red meat and sausage $(\mathrm{g} / \mathrm{d})$ & $73 \cdot 7$ & $50 \cdot 0$ & $81 \cdot 2$ & 58.4 & 63.9 & $45 \cdot 8$ & $<0.001$ \\
\hline Ratio of PUFA:SFA & 0.4 & 0.2 & 0.4 & 0.2 & 0.4 & 0.2 & 0.807 \\
\hline Total fat intake (\% energy intake) & $30 \cdot 0$ & $5 \cdot 1$ & $30 \cdot 3$ & $5 \cdot 1$ & $29 \cdot 6$ & $5 \cdot 0$ & 0.180 \\
\hline Dietary Approaches to Stop Hypertension score & $21 \cdot 0$ & 4.4 & $20 \cdot 2$ & 4.4 & $21 \cdot 7$ & 4.2 & $<0.001$ \\
\hline Fruit and fruit juices $(\mathrm{g} / \mathrm{d}) \ddagger$ & 104 & 121 & 97.9 & 117 & 113 & 124 & 0.119 \\
\hline Vegetables (potatoes excluded) (g/d) & 90.5 & $70 \cdot 0$ & $86 \cdot 7$ & $72 \cdot 0$ & 93.4 & 69.5 & 0.456 \\
\hline High-fibre $(\geq 5 \%)$ grain products $(\mathrm{g} / \mathrm{d})$ & $57 \cdot 3$ & $49 \cdot 0$ & $60 \cdot 1$ & $48 \cdot 0$ & $55 \cdot 1$ & $48 \cdot 7$ & 0.247 \\
\hline Low-fat $(<1 \%)$ milk and sour milk products $(\mathrm{g} / \mathrm{d}) \ddagger$ & 390 & 499 & 437 & 527 & 367 & 490 & 0.112 \\
\hline Red meat and sausages $(\mathrm{g} / \mathrm{d})$ & $73 \cdot 7$ & $50 \cdot 0$ & $81 \cdot 2$ & 58.4 & 63.9 & $45 \cdot 8$ & $<0.001$ \\
\hline Sugar-sweetened beverages $(\mathrm{g} / \mathrm{d}) \ddagger$ & 100 & 157 & 125 & 174 & 87.5 & 158 & 0.148 \\
\hline $\mathrm{Na}$ intake $(\mathrm{g} / \mathrm{d})$ & $2 \cdot 3$ & 0.5 & 2.5 & 0.6 & $2 \cdot 1$ & 0.4 & $<0.001$ \\
\hline Raven's Coloured Progressive Matrices score & $24 \cdot 0$ & $5 \cdot 2$ & $23 \cdot 8$ & $5 \cdot 3$ & $24 \cdot 1$ & $5 \cdot 1$ & 0.552 \\
\hline
\end{tabular}

* Data were analysed using Student's $t$ test or the Mann-Whitney $U$ test for continuous variables and using the $\chi^{2}$ test for categorical variables.

$\dagger P$ values refer to statistical significance for differences between boys and girls.

$\ddagger$ Median and interquartile range. 
Table 3. Associations of the Baltic Sea Diet Score (BSDS) and the Dietary Approaches to Stop Hypertension (DASH) score with Raven's Coloured Progressive Matrices score*

\begin{tabular}{|c|c|c|c|c|c|c|}
\hline & All $(n$ 428) & $P$ & Boys $(n 216)$ & $P$ & Girls $(n 212)$ & $P$ \\
\hline BSDS & $0.122 \dagger$ & 0.012 & $0.244 \dagger$ & $<0.001$ & -0.012 & 0.867 \\
\hline Fruit and berries $(\mathrm{g} / \mathrm{d})$ & 0.079 & 0.079 & 0.098 & 0.171 & 0.019 & 0.781 \\
\hline Vegetables (legumes included, potatoes excluded) $(\mathrm{g} / \mathrm{d})$ & 0.055 & 0.251 & $0.188 \dagger$ & 0.007 & -0.064 & 0.347 \\
\hline High-fibre $(\geq 5 \%)$ grain products $(\mathrm{g} / \mathrm{d})$ & 0.048 & 0.322 & 0.082 & 0.238 & -0.026 & 0.710 \\
\hline Low-fat $(<1 \%)$ milk $(\mathrm{g} / \mathrm{d})$ & 0.040 & 0.406 & 0.007 & 0.915 & 0.078 & 0.266 \\
\hline Fish $(g / d)$ & 0.042 & 0.381 & 0.097 & 0.158 & -0.024 & 0.725 \\
\hline Red meat and sausage $(\mathrm{g} / \mathrm{d})$ & $-0.114 \dagger$ & 0.021 & -0.126 & 0.065 & -0.069 & 0.307 \\
\hline Ratio of PUFA:SFA & 0.034 & 0.475 & 0.076 & 0.261 & 0.001 & 0.992 \\
\hline Total fat intake (\% energy intake) & 0.012 & 0.805 & -0.023 & 0.735 & 0.018 & 0.800 \\
\hline DASH score & $0.121 \dagger$ & 0.015 & $0.202 \dagger$ & 0.003 & 0.029 & 0.677 \\
\hline Fruit and fruit juice (g/d) & $0.116 \dagger$ & 0.015 & $0.179 \dagger$ & 0.009 & 0.016 & 0.816 \\
\hline Vegetables (potatoes excluded) (g/d) & 0.021 & 0.440 & $0.137 \dagger$ & 0.050 & -0.072 & 0.286 \\
\hline High-fibre $(\geq 5 \%)$ grain products $(\mathrm{g} / \mathrm{d})$ & 0.036 & 0.456 & 0.067 & 0.327 & -0.026 & 0.710 \\
\hline Low-fat $(<1 \%)$ milk and sour milk products $(\mathrm{g} / \mathrm{d})$ & 0.027 & 0.582 & 0.026 & 0.702 & 0.046 & 0.514 \\
\hline Red meat and sausage $(\mathrm{g} / \mathrm{d})$ & $-0.109 \dagger$ & 0.026 & -0.119 & 0.078 & -0.069 & 0.307 \\
\hline Sugar-sweetened beverages $(\mathrm{g} / \mathrm{d})$ & 0.091 & 0.061 & 0.104 & 0.108 & 0.042 & 0.539 \\
\hline $\mathrm{Na}$ intake $(\mathrm{mg} / \mathrm{d})$ & -0.008 & 0.882 & -0.056 & 0.420 & -0.077 & 0.254 \\
\hline
\end{tabular}

* Data are expressed as standardised regression coefficients from linear regression models adjusted for age, sex, parental education and household income.

† Values show statistically significant associations.

In the boys, the BSDS and the DASH score were directly associated with the Raven's CPM score after adjustment for age, parental education and household income (Table 3). Of the components of the BSDS, a lower intake of vegetables was related to a lower Raven's CPM score after these adjustments (Table 3). Of the components of the DASH score, a lower consumption of fruit and fruit juices and a lower consumption of vegetables were associated with a lower Raven's CPM score after adjustment for age, parental education and household income (Table 3). Further adjustments had no effect on these associations (data not shown).

In the girls, the BSDS, the DASH score or their components were not related to the Raven's CPM score (Table 3).

\section{Differences in Raven's Coloured Progressive Matrices score} among children in quartiles of Baltic Sea Diet Score and its components

Among all children, there were no differences in the Raven's CPM score among children in the quartiles of the BSDS after adjustment for age, sex, parental education and household income (Fig. 1). Children with lowest consumption of fruit and berries $(\leq 48 \mathrm{~g} / \mathrm{d}, F(3,420)=2.856, P=0.036$ for main effect) and highest consumption of red meat and sausages $(\geq 104 \mathrm{~g} / \mathrm{d}, F(3,420)=3.579, P=0.014$ for main effect) had the lowest Raven's CPM score. Further adjustments had no effect on these differences.

The Raven's CPM score increased with increasing quartiles of BSDS in boys (Fig. 1). Boys with lowest consumption of fruit and berries $(\leq 47 \mathrm{~g} / \mathrm{d}, F(3,209)=2.799, P=0.041$ for main effect), vegetables $(\leq 61 \mathrm{~g} / \mathrm{d}, \quad F(3, \quad 209)=2.682$, $P=0.048$ for main effect), high-fibre grain products $(\leq 35 \mathrm{~g} / \mathrm{d}$, $F(3,209)=4.056, \quad P=0.008$ for main effect) and fish $(\leq 4 \mathrm{~g} / \mathrm{d}, F(2,210)=3.985, P=0.020$ for main effect), and boys with highest consumption of red meat and sausages $(\geq 104 \cdot 1 \mathrm{~g} / \mathrm{d}, F(3,209)=4.329, P=0.006$ for main effect $)$ had the lowest Raven's CPM score (data not shown). Further adjustment had no effect on these differences.

In the girls, there were no differences in the Raven's CPM score among those in the quartiles of the BSDS (Fig. 1) or its components (data not shown).

Differences in Raven's Coloured Progressive Matrices score among children in quartiles of Dietary Approaches to Stop Hypertension score and the quintiles of the Dietary Approaches to Stop Hypertension score components

Children in the lowest quartile of the DASH score had a lower Raven's CPM score than children in the second quartile and in the highest quartile after adjustment for age, sex, parental education and household income (Fig. 1). Further adjustments had no effect on these differences.

Boys in the lowest quartile of the DASH score had a lower Raven's CPM score than boys in the highest quartile (Fig. 1). Boys with the lowest consumption of fruit and fruit juices $(\leq 30 \mathrm{~g} / \mathrm{d}, F(3,209)=3.988, P=0.004$ for main effect $)$ and high-fibre grain products $(\leq 46 \mathrm{~g} / \mathrm{d}, \quad F(4,208)=3.682$, $P=0.006$ for main effect) had lower Raven's CPM score than other boys. Further adjustments had no effect on these differences.

In the girls, there were no differences in the Raven's CPM score among those in the quartiles of the DASH (Fig. 1) or in the quintiles of its components (data not shown).

\section{Discussion}

We found that poor diet quality, assessed by the BSDS and the DASH score, was associated with a poorer cognition in children and especially in boys. Particularly, a low consumption of fruit and berries, and vegetables was linked to a worse cognition. Moreover, the present results provide some evidence that a lower consumption of high-fibre grain products and 

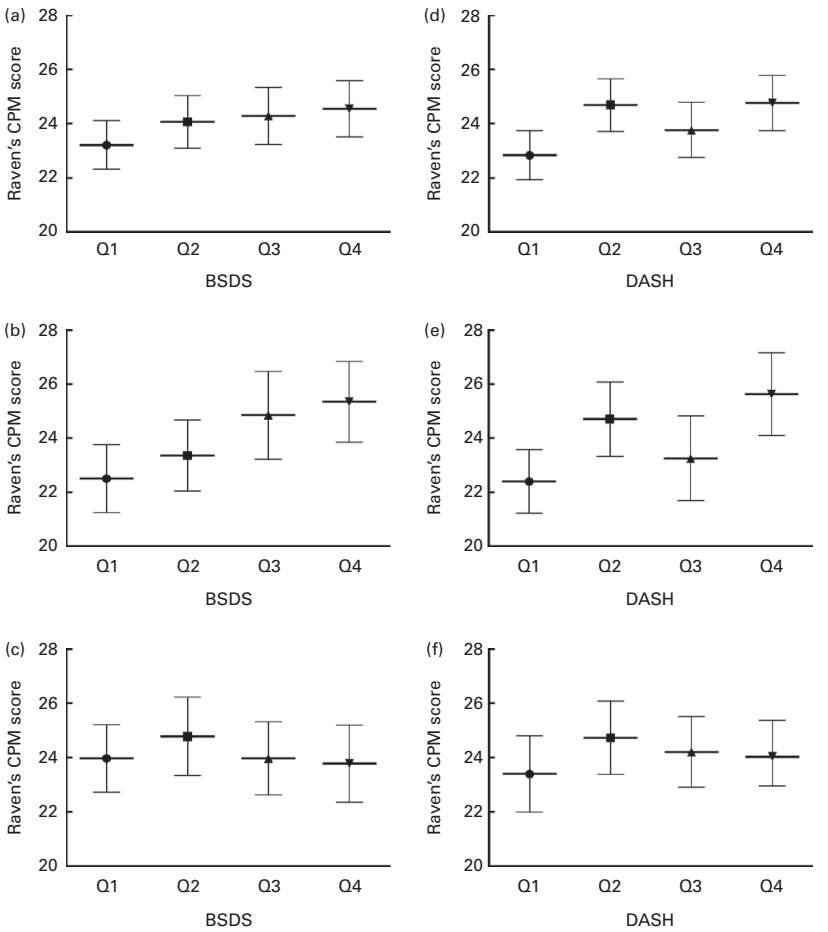

Fig. 1. Raven's Coloured Progressive Matrices (CPM) scores among 428 children (216 boys and 212 girls) in the quartiles of the Baltic Sea Diet Score (BSDS) and the Dietary Approaches to Stop Hypertension (DASH) score adjusted for age, sex, parental education and household income. Values are estimated marginal means, with $95 \%$ confidence intervals represented by vertical bars. CPM scores in quartiles of BSDS (quartile $1(\mathrm{Q} 1)=\leq 9$; quartile $2(\mathrm{Q} 2)=10-12$; quartile $3(\mathrm{Q} 3)=13-15$; quartile $4(\mathrm{Q} 4)=\geq 16)$ for (a) all children, (b) boys and (c) girls. For boys, $F(3,209)=3.397(P=0.019)$, and mean value for Q4 was significantly different from that for Q1 $(P=0.029)$. CPM scores in quartiles of DASH scores $(\mathrm{Q} 1=\leq 18 ; \mathrm{Q} 2=19-21 ; \mathrm{Q} 3=22-24$ $\mathrm{Q} 4=\geq 25$ ) for (d) all children, (e) boys and (f) girls. For all children, $F(3$, $420)=3.499(P=0.016)$, mean value for $Q 4$ was significantly different from that for Q1 $(P=0.038)$, and mean value for Q2 was significantly different from that for Q1 $(P=0.037)$. For boys, $F(3,209)=4.293(P=0.006)$, and mean value for $\mathrm{Q} 4$ was significantly different from that for $\mathrm{Q} 1(P=0.008)$.

fish and a higher consumption of red meat and sausages may be related to worse cognition. These findings are the first evidence for the associations of the BSDS and the DASH score with cognition in children.

The results of some previous studies have suggested that a poorer diet quality has been associated with a worse cognitive performance in children ${ }^{(1,14,16,38)}$. A poorer diet quality at the age of 1 year, assessed by the Eating Assessment in Toddler diet score, was linked to a worse performance in the Peabody Picture Vocabulary Test-III and the Raven's CPM at the age of 10 years $^{(16)}$. Diet low in fruits, vegetables, home-made foods, cheese and herbs and lack of breast-feeding in infancy have also been associated with a lower intelligence quotient and a poorer working memory in later years ${ }^{(38,39)}$. Moreover, a nutrient-enriched formula in the first month after birth was related to improved verbal cognitive ability at the age of 8 years in boys born preterm, but no such association had been found in girls ${ }^{(40)}$. These studies have focused on the associations of dietary factors in infancy with cognition later in life; however, there are few studies on this issue in school-aged children. However, a better diet quality, assessed by the Healthy Eating Index, had been associated with a better cognitive control in one cross-sectional study among children 8 years of age ${ }^{(7)}$

The present study showed that the BSDS and the DASH score had stronger direct associations with cognition than their components, suggesting that the diet as a whole is a better predictor of cognition than single foods or nutrients. Cognition was poorest among children who were in the lowest quartile of the BSDS or the DASH score. Cognition also increased in a dose-dependent manner with increasing BSDS score, whereas the association between the DASH score and cognition was less linear. This finding suggests that the BSDS was a slightly better predictor for cognition than the DASH score in the present study sample of children. Nevertheless, our observations support the earlier findings that a diet high in fruit, berries and vegetables is beneficial for cognition in children ${ }^{(36,38)}$. Moreover, a higher consumption of red meat and sausages and a lower consumption of fish may be harmful for cognition in children.

There are a few possible explanations for our findings. A diet low in fruit, vegetables and fish and high in SFA has been associated with increased cardiometabolic risk ${ }^{(41)}$ that has been linked to smaller hippocampal volumes, signs of frontal lobe atrophy and a worse cognition in adolescents ${ }^{(42)}$. Vitamins, polyphenols and flavonoids found in fruit, berries and vegetables may protect the brain against neuronal damage by decreasing inflammation and oxidative stress and by supporting cell proliferation ${ }^{(43)}$. Moreover, a high intake of SFA may decrease and a high intake of flavonoids found in fruits, berries and vegetables may increase circulating concentrations of brain-derived neurotrophic factor ${ }^{(43,44)}$. Brain-derived neurotrophic factor is a growth factor that has been shown to enhance synaptic plasticity, neurogenesis, neural survival, learning and memory ${ }^{(43,44)}$. Moreover, the intake of DHA, which is found in fish, has been directly associated with endothelial NO synthesis and may thereby dilate arteries and increase cerebral blood flow ${ }^{(43)}$.

There is no clear reason for our observation that the associations of dietary factors with cognition were stronger in boys than in girls. The boys had a lower DASH score and a slightly lower BSDS than the girls. They also had a higher consumption of red meat and sausages, and a higher $\mathrm{Na}$ intake than the girls. However, there was no difference in the Raven's CPM score between sexes that makes it unlikely that different distributions of variables would explain the findings. There is some evidence that male brains are more vulnerable to stress than female brains ${ }^{(45)}$ and that boys' cognitive development benefit more from dietary intervention during infancy than that of girls ${ }^{(39)}$. Moreover, nutrient-enriched formula during infancy had been associated with increased caudate nucleus volumes at the age of 15 years in boys but not in girls ${ }^{(46)}$. Frontal and parietal cortices of the brain reach their peak thickness 1 year later in boys than in girls ${ }^{(47)}$. It is, therefore, possible that the later maturation of male brains could partly explain the stronger association of dietary factors with cognition in boys than in girls in the present study sample ${ }^{(47,48)}$. However, there are few studies in which the associations of 
dietary factors with cognition would have been investigated in girls and boys separately, and the results of those studies are equivocal $^{(49,50)}$. Therefore, more studies on sex differences in the associations of dietary factors with cognition are highly warranted.

The strengths of the present study include a relatively large population-based sample of children and the rigorous methods used for assessing dietary patterns, their components and cognition. We also had an opportunity to control the data for a number of possible confounding factors, including skipping meals and eating behaviour, in the analyses. The ethnic background and place of residence of the children were homogenous, and none of the children had consumed alcohol or smoked cigarettes that makes them as unlikely confounding factors. One weakness of the present study is the crosssectional design that does not allow us to make conclusions about the causality of the relationships. Moreover, we did not assess the latest meals before the assessment of cognition that could have had an effect on the Raven's CPM score in children $^{(51)}$

The results of the present study suggest that a poorer diet quality is associated with a worse cognition in children and that the relationships are much stronger in boys than in girls. These findings emphasise a diet high in fruit, berries, vegetables and fish and low in red meat and sausages in order to support the normal development of cognition among children.

\section{Acknowledgements}

The present study was funded by the Ministry of Social Affairs and Health of Finland, Ministry of Education and Culture of Finland, University of Eastern Finland, Finnish Innovation Fund Sitra, Social Insurance Institution of Finland, Finnish Cultural Foundation, Juho Vainio Foundation, Foundation for Paediatric Research, Paavo Nurmi Foundation, Paulo Foundation, Diabetes Research Foundation, Research Committee of the Kuopio University Hospital Catchment Area (State Research Funding) and Kuopio University Hospital (EVO funding number 5031343), Päivikki and Sakari Sohlberg Foundation, City of Kuopio.

The authors' contributions are as follows: E. A. H., A.-M. E., V. L. and T. A. L. designed the study; A.-M. E., T. V., U. S., V. L. and T. A. L. conducted the study; E. A. H. analysed the data; E. A. H., A.-M. E., T. V., U. S., V. L. and T. A. L. wrote the manuscript; E. A. H., A.-M. E. and T. A. L. had primary responsibility for the final content of the manuscript. All authors read and approved the final version of the manuscript.

None of the authors has any conflicts of interest to declare.

\section{References}

1. Nyaradi A, Li J, Hickling S, et al. (2013) The role of nutrition in children's neurocognitive development, from pregnancy through childhood. Front Hum Neurosci 7, 97.

2. Burkhalter TM \& Hillman CH (2011) A narrative review of physical activity, nutrition, and obesity to cognition and scholastic performance across the human lifespan. Adv Nutr 2, 201-206.

3. Taras H (2005) Nutrition and student performance at school. J Sch Health 75, 199-213.

4. Féart C, Samieri C, Allès B, et al. (2013) Potential benefits of adherence to the Mediterranean diet on cognitive health. Proc Nutr Soc 72, 140-152.

5. Baym CL, Khan NA, Monti JM, et al. (2014) Dietary lipids are differentially associated with hippocampal-dependent relational memory in prepubescent children. Am J Clin Nutr 99, 1026-1032.

6. Zhang J, Hebert J \& Muldoon M (2005) Dietary fat intake is associated with psychosocial and cognitive functioning of school-aged children in the United States. J Nutr 135, 1967-1973.

7. Khan NA, Raine LB, Drollette ES, et al. (2015) Dietary fiber is positively associated with cognitive control among prepubertal children. J Nutr 145, 143-149.

8. Diamond A (2013) Executive functions. Annu Rev Psychol 64, 135-168.

9. Tangney CC \& Scarmeas N (2012) The good, bad, and ugly? How blood nutrient concentrations may reflect cognitive performance. Neurology 78, 230-231.

10. Allès B, Samieri C, Féart C, et al. (2012) Dietary patterns: a novel approach to examine the link between nutrition and cognitive function in older individuals. Nutr Res Rev 25 , 207-222.

11. Sacks FM, Obarzanek EVA, Windhauser MM, et al. (1995) Rationale and design of the Dietary Approaches to Stop Hypertension trial (DASH). A multicenter controlled-feeding study of dietary patterns to lower blood pressure. Ann Epidemiol 5, 108-118.

12. Wengreen H, Munger R, Cutler A, et al. (2013) Prospective study of Dietary Approaches to Stop Hypertension- and Mediterranean-style dietary patterns and age-related cognitive change: the Cache County Study on memory, health and aging. Am J Clin Nutr 98, 1263-1271.

13. Kesse-guyot E, Andreeva VA, Lassale C, et al. (2013) Mediterranean diet and cognitive function?: a French study. $A m J$ Clin Nutr 97, 369-376.

14. Vassiloudis I, Yiannakouris N, Panagiotakos DB, et al. (2014) Academic performance in relation to adherence to the Mediterranean diet and energy balance behaviors in Greek primary schoolchildren. J Nutr Educ Behav 46, 164-170.

15. Kanerva N, Kaartinen NE, Schwab U, et al. (2013) Adherence to the Baltic Sea diet consumed in the Nordic countries is associated with lower abdominal obesity. Br J Nutr 109, $520-528$.

16. Nyaradi A, Li J, Hickling S, et al. (2013) Diet in the early years of life influences cognitive outcomes at 10 years: a prospective cohort study. Acta Paediatr 102, 1165-1173.

17. Eloranta A-M, Lindi V, Schwab U, et al. (2012) Dietary factors associated with overweight and body adiposity in Finnish children aged 6-8 years: the PANIC Study. Int J Obes 36, 950-955.

18. National Public Health Institute (2006) Picture Book of Food Portion Sizes. Helsinki, Finland: The National Public Health Institute.

19. Rastas M, Seppänen R, Knuts L, et al. (1997) Nutrient Composition of Foods. Helsinki, Finland: The Social Insurance Institution.

20. Wardle J, Guthrie CA, Sanderson S, et al. (2001) Development of the children's eating behaviour questionnaire. J Child Psychol Psychiatry 42, 963-970. 
21. Raven J, Raven J \& Court J (1998) Coloured Progressive Matrices. Manual for Raven's Progressive Matrices and Vocabulary Scales. London: Oxford Psychologist Press Ltd.

22. Lintu N, Tompuri T, Viitasalo A, et al. (2014) Cardiovascular fitness and haemodynamic responses to maximal cycle ergometer exercise test in children 6-8 years of age. J Sports Sci 32, 652-659.

23. Haapala EA, Poikkeus A-M, Tompuri T, et al. (2014) Associations of motor and cardiovascular performance with academic skills in children. Med Sci Sports Exerc 46, $1016-1024$

24. Väistö J, Eloranta A-M, Viitasalo A, et al. (2014) Physical activity and sedentary behaviour in relation to cardiometabolic risk in children: cross-sectional findings from the Physical Activity and Nutrition in Children (PANIC) Study. Int J Behav Nutr Phys Act 11, 55.

25. Haapala EA, Poikkeus A-M, Kukkonen-Harjula $\mathrm{K}$, et al. (2014) Associations of physical activity and sedentary behavior with academic skills - a follow-up study among primary school children. PLOS ONE 10, e107031.

26. Saari A, Sankilampi U, Hannila M-L, et al. (2011) New Finnish growth references for children and adolescents aged 0 to 20 years: length/height-for-age, weight-for-length/height, and body mass index-for-age. Ann Med 43, 235-248.

27. Cole T, Bellizzi M, Flegal K, et al. (2000) Establishing a standard definition for child overweight and obesity worldwide: international survey. BMJ 320, 1240-1243.

28. Tompuri TT, Lakka TA, Hakulinen M, et al. (2015) Assessment of body composition by dual-energy X-ray absorptiometry, bioimpedance analysis and anthropometrics in children: the Physical Activity and Nutrition in Children study. Clin Physiol Funct Imaging 35, 21-33.

29. Tanner J (1962) Growth at Adolescence. Oxford: Blackwell.

30. Malina RM, Bouchard C \& Bar-Or O (2004) Growth, Maturation, and Physical Activity, 2nd ed. Champaign: Human Kinetics.

31. Fung TT, Chiuve SE, Mccullough ML, et al. (2008) Adherence to a DASH-style diet and risk of coronary heart disease and stroke in women. Arch Intern Med 168, 713-720.

32. Sirin SR (2005) Socioeconomic status and academic achievement: a meta-analytic review of research. Rev Educ Res 75, 417-453.

33. Hillman CH, Pontifex MB, Castelli DM, et al. (2014) Effects of the FITKids randomized controlled trial on executive control and brain function. Pediatrics 134, e1063-e1071.

34. Haapala EA (2013) Cardiorespiratory fitness and motor skills in relation to cognition and academic performance in children - a review. J Hum Kinet 36, 55-68.

35. Reinert KRS, Po'e EK \& Barkin SL (2013) The relationship between executive function and obesity in children and adolescents: a systematic literature review. J Obes 2013, 820956.
36. Jernigan T, Baare W, Stiles J, et al. (2011) Postnatal brain development: structural imaging of dynamic neurodevelopmental processes. Prog Brain Res 189, 77-92.

37. Diamond A (2000) Close interrelation of motor development and cognitive development and of the cerebellum and prefrontal cortex. Child Dev 71, 44-56.

38. Smithers LG, Golley RK, Mittinty MN, et al. (2013) Do dietary trajectories between infancy and toddlerhood influence IQ in childhood and adolescence? Results from a prospective birth cohort study. PLOS ONE 8, e58904.

39. Gale CR, Martyn CN, Marriott LD, et al. (2009) Dietary patterns in infancy and cognitive and neuropsychological function in childhood. I Child Psychol Psychiatry 50, $816-823$

40. Lucas A, Morley R \& Cole TJ (1998) Randomised trial of early diet in preterm babies and later intelligence quotient. BMJ 317, 1481-1487.

41. Hu FB, Rimm EB, Stampfer MJ, et al. (2000) Prospective study of major dietary patterns and risk of coronary heart disease in men. Am J Clin Nutr 72, 912-921.

42. Yates KF, Sweat V, Yau PL, et al. (2012) Impact of metabolic syndrome on cognition and brain: a selected review of the literature. Arterioscler Thromb Vasc Biol 32, 2060-2067.

43. Frisardi V, Panza F, Seripa D, et al. (2010) Nutraceutical properties of Mediterranean diet and cognitive decline: possible underlying mechanisms. J Alzheimer's Dis 22, 715-740.

44. Wu A, Ying Z \& Gomez-Pinilla F (2004) The interplay between oxidative stress and brain-derived neurotrophic factor modulates the outcome of a saturated fat diet on synaptic plasticity and cognition. Eur $J$ Neurosci 19 1699-1707.

45. Markham JA, Mullins SE \& Koenig JI (2013) Periadolescent maturation of the prefrontal cortex is sex-specific and is disrupted by prenatal stress. J Comp Neurol 521, 1828-1843.

46. Isaacs EB, Gadian DG, Sabatini S, et al. (2008) The effect of early human diet on caudate volumes and IQ. Pediatr Res 63, 308-314.

47. Patton G \& Viner R (2007) Adolescent health 1: pubertal transitions in health. Lancet 369, 1130-1139.

48. Knudsen EI (2004) Sensitive periods in the development of the brain and behavior. $J$ Cogn Neurosci 16, 1412-1425.

49. Lassek WD \& Gaulin SJC (2011) Sex differences in the relationship of dietary fatty acids to cognitive measures in American children. Front Evol Neurosci 3, 1-8.

50. Stea TH \& Torstveit MK (2014) Association of lifestyle habits and academic achievement in Norwegian adolescents: a cross-sectional study. BMC Public Health 14, 829.

51. Adolphus K, Lawton CL \& Dye L (2013) The effects of breakfast on behavior and academic performance in children and adolescents. Front Hum Neurosci 7, 425. 